\title{
Clinical Impact of Targeting Inaccuracy Due to Distortion of the Leksell Stereotactic Frame
}

\author{
Cécile Renier ${ }^{1}$, Nicolas Massager ${ }^{2 *}$ \\ ${ }^{1}$ Department of Radiation Physics, Institute Jules Bordet, Brussels, Belgium \\ ${ }^{2}$ Department of Neurosurgery, University Hospital Tivoli, La Louvière, Belgium \\ Email: cecile.renier@bordet.be, *nicolas.massager@ulb.ac.be
}

How to cite this paper: Renier, C. and Massager, N. (2018) Clinical Impact of Targeting Inaccuracy Due to Distortion of the Leksell Stereotactic Frame. Open Journal of Modern Neurosurgery, 8, 315-322. https://doi.org/10.4236/ojmn.2018.83026

Received: May 28, 2018

Accepted: July 21, 2018

Published: July 24, 2018

Copyright $(0) 2018$ by authors and Scientific Research Publishing Inc. This work is licensed under the Creative Commons Attribution International License (CC BY 4.0).

http://creativecommons.org/licenses/by/4.0/

\begin{abstract}
Background: Distortion of the Leksell stereotactic frame can occur during surgical and radiosurgical frame-based procedures. The targeting accuracies of surgical procedures such as DBS surgery or frame-based biopsy, or Gamma Knife radiosurgical procedures, are related to the stereotactic frame that is commonly referred to a non-deformable referential system. Objective: To evaluate the clinical impact of frame distortion on accuracy of targeting in various stereotactic procedures. Methods: We studied the influence of increasing levels of distortion of the Leksell frame both on surgical procedures using the stereotactic arch, and on radiosurgical procedures with the Gamma Knife. For surgical procedures we applied the Target Simulator of Elekta to a frame submitted to different levels of frame distortion, and we measured the modifications of accuracy of targeting. For radiosurgical procedures the Leksell frame was applied on an anthropomorphic phantom and modifications of target coordinates were measured by an imaging method using the stereotactic CBCT of the Gamma Knife ICON. Results: For surgical procedures, we found a linear relation between the amount of the frame distortion and the extent of the deviation from the stereotactic target using the stereotactic arch $\left(\mathrm{R}^{2}=0.99709\right)$. The level of bending of the frame is also linearly related to the level of inaccuracy of stereotactic targeting based on acquisition of MRI and CTscan with the Elekta imaging boxes $\left(R^{2}=0.96825\right)$. The inaccuracy of targeting related to frame bending can be avoided by a systematic control at the end of frame placement and by the use of the CBCT of the Gamma Knife Icon. Conclusion: Distortion of the frame is a significant source of clinical inaccuracy of targeting for stereotactic procedures. Frame bending must be checked after each frame placement and corrected if needed to avoid targeting inaccuracy during stereotactic surgery and radiosurgery procedures.
\end{abstract}

\section{Keywords}

Targeting, Inaccuracy, Distortion, Frame, Stereotactic 


\section{Introduction}

We have observed, as other, in our clinical daily practice that the pressure exerted on the fixation screws to secure the frame on the patient's head can create a significant distortion of the Leksell head frame [1] [2]. The pressures used in daily clinical practice may induce such significant frame bending. Yet, the real clinical impact of distortion of the stereotactic frame remains unknown. Does frame bending really induce a problematic inaccuracy in stereotactic targeting for surgical procedures such as DBS surgery or frame-based biopsy, or for Gamma Knife radiosurgical procedures? Different referential systems were applied on the frame for these procedures. Moreover, the fixation of various stereotactic instruments (stereotactic arc for surgical procedures, frame adapter for Gamma Knife) to the frame could probably influence in different ways the consequences of frame distortion on the accuracy of targeting.

The aim of our work was to study the clinical impact of targeting inaccuracy produced by distortions of the Leksell frame when applied in various stereotactic conditions.

\section{Materials and Methods}

The present study was approved by the local Institutional Review Board of the Ethical Committee of our institution (ref. P2015/207). Informed consent of all patients included in this study was obtained.

Our analysis has been focused on two different stereotactic applications of the Leksell G frame.

\subsection{Surgical Procedures}

The first experiment would study the effect of frame distortion on targeting in 60 surgical procedures performed with the Leksell stereotactic arch applied on the frame. For this purpose, we have used the Target Simulator of Elekta ${ }^{\oplus}$ (Elekta Instruments, Sweden). We applied the Target Simulator on a stereotactic frame not strained by any significant pressure. We fixed the stereotactic arch on the frame and we used a Sedan biopsy needle of $2.5 \mathrm{~mm}$ diameter to reach the target (Figure 1, upper left \& middle). We put the stereotactic coordinates on the frame. We observed that the needle has reached the target with a inframillimetric precision. Then, we mounted at the reverse part of the frame a rigid sphere of $15 \mathrm{~cm}$ diameter tightened with 4 fixation screws. We used a low pressure to secure the sphere and we observed that the biopsy needle still reached the stereotactic target with a inframillimetric precision (Figure 1, lower left). Then, we applied different conditions of pressure in order to create a distortion of the frame, and we measured the modifications of accuracy of targeting of the biopsy needle in relation to the target provided by the Target Simulator (Figure 1, lower right).

\subsection{Gamma Knife Procedures}

The second experimental study focused the influence of distortion of the Leksell 


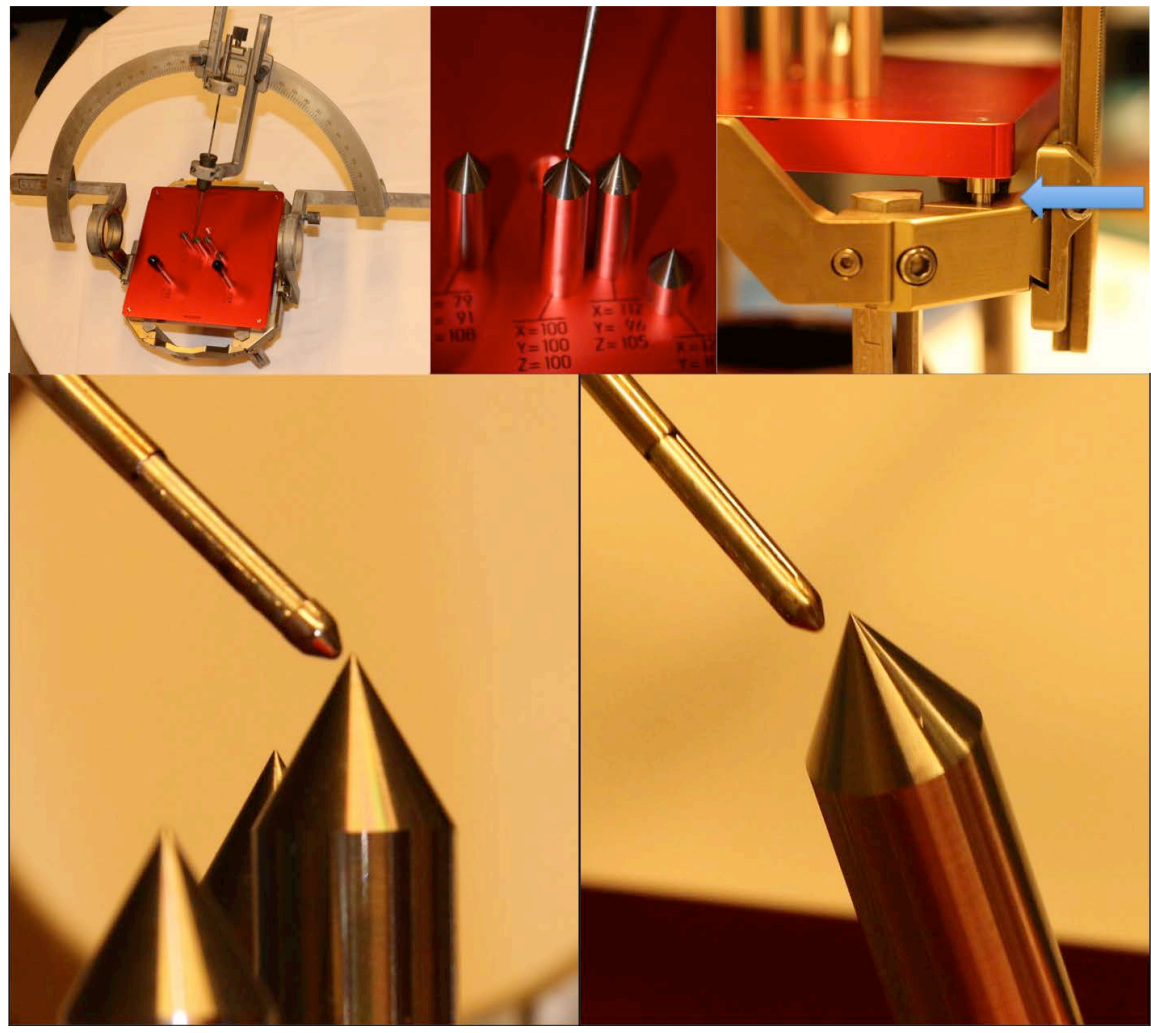

Figure 1. Use of the Target Simulator of Elekta ${ }^{\oplus}$ to study the effect of frame distortion on targeting of surgical procedures using the stereotactic arch applied on the frame. Lower left: using a low pressure, the biopsy needle reached the stereotactic target. Lower right: using a high pressure in order to create a distortion of the frame, inaccuracy of targeting of the biopsy needle.

frame on the radiation targeting of the Leksell Gamma Knife Icon ${ }^{\circledR}$ (Elekta Instruments, Sweden). We placed the Leksell frame on the head of an anthropomorphic phantom and we applied different conditions of pressure in order to create increasing distortions of the stereotactic frame. A series of 30 measurements were performed. We evaluated the targeting inaccuracy induced by distortion of the frame with the following method: we compared the stereotactic coordinates of a target located in the head issued from 2 different stereotactic CTscan. The first one was the conventional stereotactic 1-mm 3D CTscan acquired after having placed the CT Indicator box of Elekta ${ }^{\oplus}$ (Elekta Instruments, Sweden) on the frame, as used in a clinical routine. The location of the fiducial markers sets on the tomographic images was used by the Stereotactic Reference Definition program of Leksell GammaPlan 11.0 to define the stereotactic coordinates of tomographic images. The indicator box is docked on the frame; so, bending of the frame will unavoidably influence the stereotactic coordinates issued from the box. The second stereotactic CTscan was the stereotactic Cone Beam CT (CBCT) of the Gamma Knife Icon [3] [4]. The acquisition of stereotactic coordinates from the $\mathrm{CBCT}$ is independent of any CT indicator box and is directly provided by the Gamma Knife ICON system once the head frame has been docked on the couch with the frame adaptor. So, the CBCT-related stereo- 
tactic coordinates are independent of frame bending. We analysed the difference of stereotactic coordinates of the target provided by these 2 imaging methods, which will represent the inaccuracy of targeting induced by frame distortion in real clinical conditions. The ICON procedure using CBCT as reference will give corrections of coordinates in the 3 directions and vectorial correction that must be applied to correct the inaccuracy of targeting [3] [4]. In order to confirm the accuracy of the correction applied, we used gafchromic films to irradiate the target without and with the correction proposed by the Gamma Knife ICON.

\section{Results}

\subsection{Surgical Procedures}

We placed the Target Simulator on the stereotactic frame strained by different pressures to create 6 values of frame bending: $0.5 \mathrm{~mm}, 1.0 \mathrm{~mm}, 1.5 \mathrm{~mm}, 2.0 \mathrm{~mm}$, $2.5 \mathrm{~mm}$ and $3.0 \mathrm{~mm}$. We measured the distance of the tip of the stereotactic cannula from the target. Les values recorded were respectively $0.3 \mathrm{~mm}, 0.8 \mathrm{~mm}$, $1.1 \mathrm{~mm}, 1.5 \mathrm{~mm}, 1.8 \mathrm{~mm}$ and $2.2 \mathrm{~mm}$ (Figure 2). The trend line has a $\mathrm{R}^{2}$ of 0.99709 . The equation of the trend line was $\mathrm{y}=0.7357 \mathrm{x}+0.036$.

\subsection{Gamma Knife Procedures}

We performed measurements of the variation between the stereotactic coordinates of the CT-related targeting and CBCT-related targeting, when the frame was not distorted and with 5 levels of frame distortions at $0.5 \mathrm{~mm}, 0.85 \mathrm{~mm}, 1.0$ $\mathrm{mm}, 1.5 \mathrm{~mm}$ and $2.0 \mathrm{~mm}$ (Table 1). Without frame distortion, the difference in stereotactic coordinates was a translation of $-0.31 \mathrm{~mm}$ in X-axis, -0.55 in $\mathrm{Y}$-axis, and -0.18 in $\mathrm{Z}$-axis. When increasing distortions were applied to the frame, the differences in stereotactic coordinates were increasing, as shown in Figure 3. The vectorial deviation was $0.66 \mathrm{~mm}$ when the frame was not bent, and increased to a maximum of $2.12 \mathrm{~mm}$ with increasing frame distortion. The trend line (Figure 3 ) has a $\mathrm{R}^{2}$ of 0.96825 . The equation of the trend line is $\mathrm{y}=$ $0.6721 x+0.7299$.

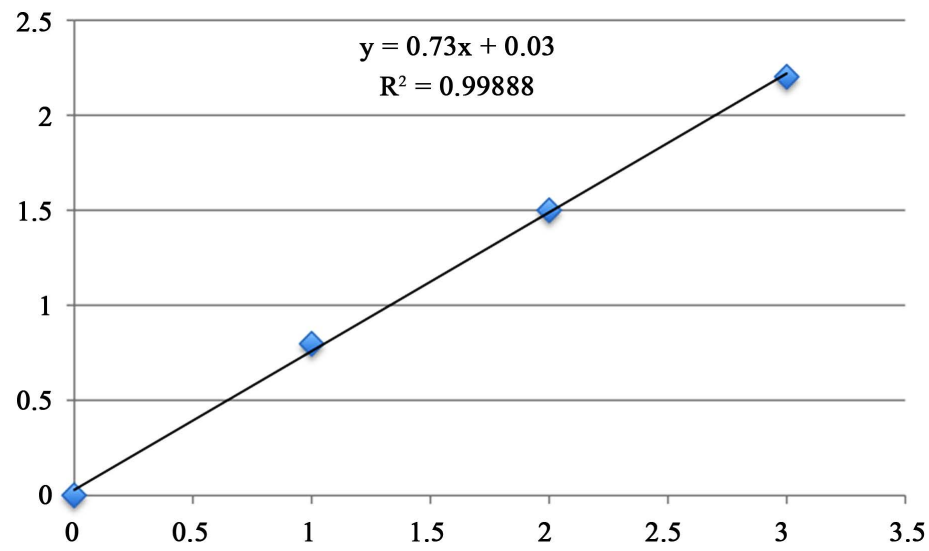

Figure 2. Graph showing the relation between frame distortion (X-axis, in $\mathrm{mm}$ ) and targeting inaccuracy ( $\mathrm{Y}$-axis, in $\mathrm{mm}$ ) with the Target Simulator model. 


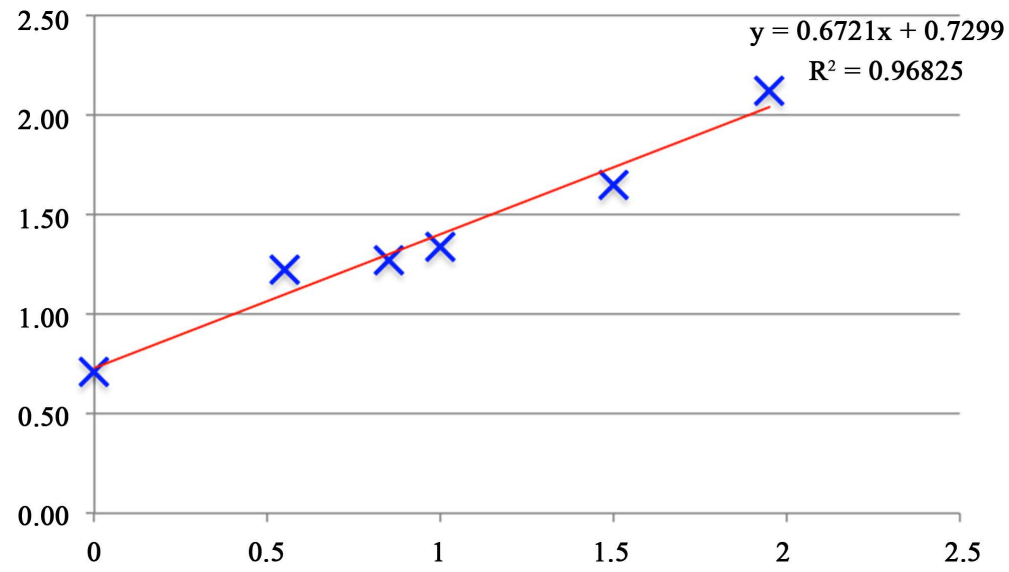

Figure 3. Graph showing the relation between frame distortion (X-axis, in $\mathrm{mm}$ ) and corrections of the stereotactic coordinates provided by the CBCT-related ICON procedure (Y-axis, in $\mathrm{mm})$.

Table 1. Corrections of the stereotactic coordinates provided by the CBCT-related ICON procedure when different levels of frame distortion are applied.

\begin{tabular}{ccccc}
\hline DISTORTION & X-axis & Y-axis & Z-axis & Vectorial \\
\hline 0 & -0.63 & -0.31 & -0.10 & 0.71 \\
0.5 & -1.21 & -0.18 & 0.03 & 1.22 \\
0.85 & -1.24 & -0.26 & 0.10 & 1.27 \\
1.0 & -1.33 & -0.09 & 0.13 & 1.34 \\
1.5 & -1.63 & -0.03 & 0.24 & 1.65 \\
2.0 & 2.08 & -0.02 & 0.41 & 2.12 \\
\hline
\end{tabular}

Analysis of the gafchromic films of the irradiated target obtained after that the correction proposed by the Gamma Knife ICON system has not or has been applied, confirmed the accuracy of the correction applied. The Figure 4 shows gafchromic films after irradiation of the target when the stereotactic coordinates from the CT indicator box were used with a frame not distorted (left figure), when the stereotactic coordinates from the CT indicator box were used with a frame distorted by $3.0 \mathrm{~mm}$ (median figure), and when the stereotactic coordinates from the CBCT of the ICON procedure were used with a frame distorted by $3.0 \mathrm{~mm}$ (right figure). The target was reached with a high accuracy when the frame was not bent (left figure). With a frame distorted by $3.0 \mathrm{~mm}$, the target was significantly shifted when stereotactic coordinates from the CT indicator box were used (median figure), and was reached with a high accuracy when the stereotactic coordinates from the CBCT of the ICON procedure were used (right figure). Figure 5 shows dose profile analyses of the films using stereotactic coordinates from the CT indicator box (left figure) and using stereotactic coordinates from the CBCT of the ICON procedure (right figure). The stereotactic coordinates of the target were shifted by $2.635 \mathrm{~mm}$ on the dose profile of the films using stereotactic coordinates from the CT indicator box. 


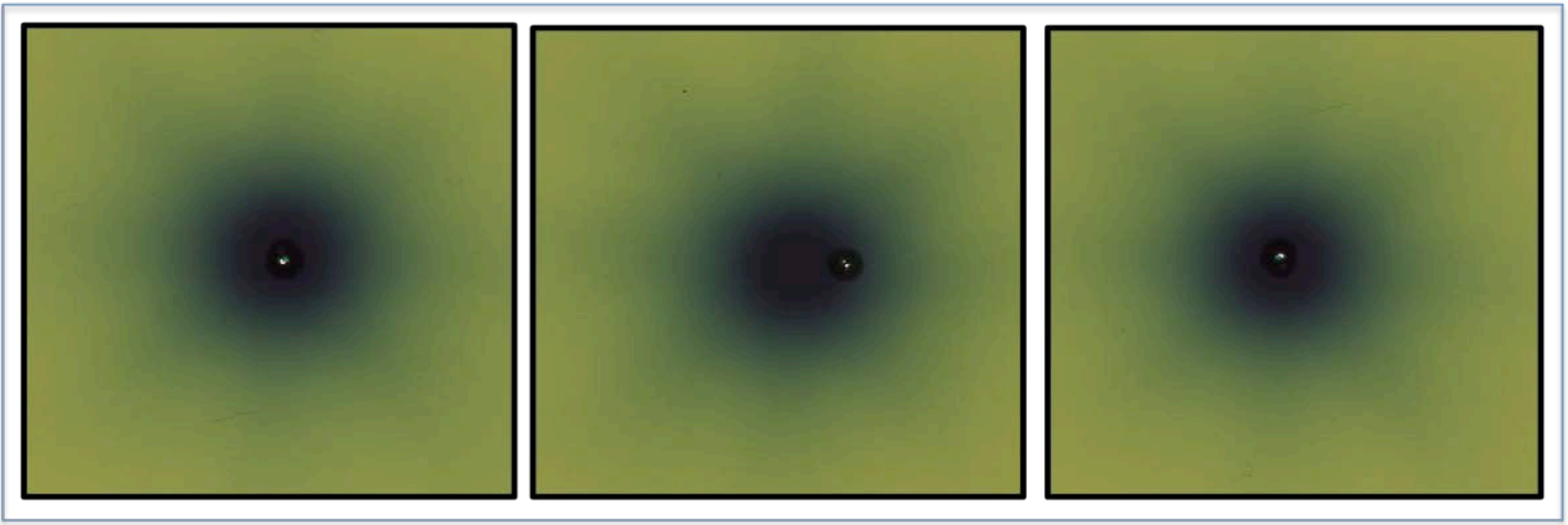

Figure 4. Gafchromic films after irradiation of the target when the stereotactic coordinates from the CT indicator box were used with a frame not distorted (left), when the stereotactic coordinates from the CT indicator box were used with a frame distorted by $3.0 \mathrm{~mm}$ (median), and when the stereotactic coordinates from the CBCT of the ICON procedure were used with a frame distorted by $3.0 \mathrm{~mm}$ (right). The target was reached with a high accuracy when the frame was not bent (left). With a frame distorted by 3.0 $\mathrm{mm}$, the target was significantly shifted when stereotactic coordinates from the CT indicator box were used (median), and was reached with a high accuracy when the stereotactic coordinates from the CBCT of the ICON procedure were used (right).

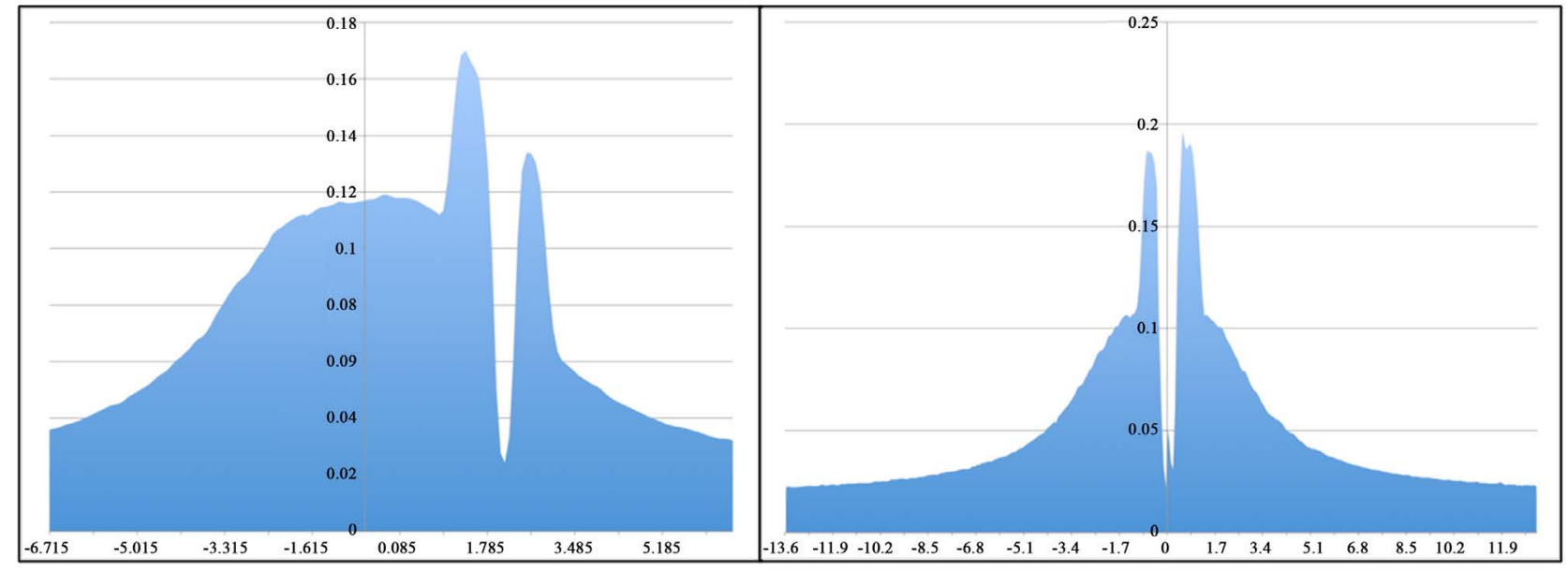

Figure 5. Dose profile analyses of the films using stereotactic coordinates from the CT indicator box (left) and using stereotactic coordinates from the CBCT of the ICON procedure (right). The stereotactic coordinates of the target were shifted by $2.635 \mathrm{~mm}$ on the dose profile of the films using stereotactic coordinates from the CT indicator box. X-axis: measurement of the deviation of the stereotactic X-coordinate (in $\mathrm{mm}$ ) from the center of the radiation target. Y-axis: radiation dose (in Gy).

\section{Discussion}

The stereotactic frame is widely used for surgical or radiosurgical procedures when a high accuracy of targeting is expected. Our practice as well as some experimental published studies [1] [2] have shown that the Leksell stereotactic frame is bendable under certain conditions that may occur in daily clinical use. The consequences of frame distortion in terms of inaccuracy of targeting are not well known, although is represents a highly critical information for the quality of stereotactic frame-based surgical and radiosurgical procedures.

The results of our experience using the Target Simulator of Elekta as experimental model of frame distortion have shown that distortion of the Leksell 
frame has a direct and significant impact on the accuracy of targeting for stereotactic procedures using the stereotactic arch. The fixations of the stereotactic arch applied on both sides of the frame are in their turn distorted by frame bending, which will as a consequence influence the accuracy of targeting of the stereotactic instruments. We found a linear relation between the amount of frame distortion and the extent of deviation from the target.

We have performed some experiments to evaluate the accuracy of the stereotactic coordinates issued from CT and MR acquisitions when the Leksell stereotactic frame is subjected to some distortion constraint. We have demonstrated that frame distortion will affect significantly the accuracy of the CT- and MR-based stereotactic coordinates. The MR and CT indicator boxes are applied on the frame and when the frame is bent, the imaging series will provide inaccurate stereotactic coordinates. There is a linear relation between the importance of frame distortion and the level of deviation of the stereotactic coordinates issued from imaging acquisition.

For surgical procedures using the Leksell stereotactic arch, the two sources of inaccuracy in targeting will occur: the stereotactic error from imaging acquisition is added to the stereotactic error from fixation of the stereotactic arch to the bended frame. For Gamma Knife radiosurgical procedures, only the error from imaging acquisition will occur.

Preventive measures can be taken to avoid distortion of the Leksell frame during fixation on the patient's head. The first one is to follow the instructions for use provided by Elekta [5]. However, we have experienced that the frame can bend in daily clinical use even when recommendations for placement are correctly followed. Therefore, we used now an undeformable box that is placed at the end of application of the frame on patient's head to check for frame distortion; when the frame is distorted, we modify the conditions of pressure on the 4 screws to correct it.

For radiosurgical procedures, the used the Gamma Knife Icon ${ }^{\oplus}$ to adjust stereotactic coordinates when the frame and frame-based imaging acquisitions have some sources of errors, as frame distortion. The stereotactic CBCT that can be acquired before irradiation gives 3D CT images that can be used to obtain stereotactic coordinates of the target independently of the frame [3] [4]. Our experiment using gafchromic films has confirmed that this procedure is a valuable issue to overcome the problem of inaccuracy related to frame distortion.

\section{Conclusion}

Distortion of the Leksell frame is a source of inaccuracy of frame-based coordinates of stereotactic targets for surgical or radiosurgical procedures. The amount of deviation from the target is related to the level of frame bending. Image acquisition with the CT- or MR-box and fixation of the stereotactic arch on a distorted frame represent the two sources of inaccuracy in stereotactic targeting. The use of an undeformable box at the end of application of the frame and use of 
the CBCT of the Gamma Knife Icon can avoid the consequences of frame distortion as a source of targeting inaccuracy in clinical use.

\section{Conflict of Interest Statement}

The authors report no conflict of interest concerning the materials or methods used in this study or the findings specified in this paper.

\section{Disclosure of Funding Statement}

No grant or any source of financial or material support was received for this study.

No any portion of this study has been presented or published previously.

\section{References}

[1] Maciunas, R.J., Galloway, R.L. and Latimer, J.W. (1994) The Application Accuracy of Stereotactic Frames. Neurosurgery, 35, 682-695. https://doi.org/10.1227/00006123-199410000-00015

[2] Treuer, H., Hunsche, S., Hoevels, M., et al. (2004) The Influence of Head Frame Distortions on Stereotactic Localization and Targeting. Physics in Medicine \& Biology, 49, 3877-3887. https://doi.org/10.1088/0031-9155/49/17/004

[3] Li, W., Bootsma, G., Von Schultz, O., Carlsson, P., Lapierre, N., Millar, B.A. and Chung, C. (2016) Preliminary Evaluation of a Novel Thermoplastic Mask System with Intra-Fraction Motion Monitoring for Future Use with Image-Guided Gamma Knife. Cureus, 8, e531. https://doi.org/10.7759/cureus.531

[4] AlDahlawi, I., Prasad, D. and Podgorsak, M.B. (2017) Evaluation of Stability of Stereotactic Space Defined by Cone-Beam CT for the Leksell Gamma Knife Icon. Journal of Applied Clinical Medical Physics, 18, 67-72. https://doi.org/10.1002/acm2.12073

[5] Elekta Instrument, A.B. (2015) Leksell Stereotactic System ${ }^{\circledR}$ Instructions for Use. 1007063 Rev. 04, Stockholm, Sweden. 